\title{
HIV-associated neurocognitive disorders: Antiretroviral regimen, central nervous system penetration effectiveness, and cognitive outcomes
}

\author{
H M Cross, ${ }^{1}$ MB ChB, Dip HIV Man (SA), MSc (Med) (Neurosci); M I Combrinck, ${ }^{1,2}$ MB ChB, BSc (Med) Hons, PhD, FCP (SA) (Neurol), MRCP; \\ J A Joska, ${ }^{3} \mathrm{MB}$ ChB, MMed (Psych), FC Psych (SA), PhD \\ ${ }^{1}$ Division of Neurology, Department of Medicine, University of Cape Town and Groote Schuur Hospital, Cape Town, South Africa \\ ${ }^{2}$ Division of Geriatric Medicine, Department of Medicine, University of Cape Town and Groote Schuur Hospital, Cape Town, South Africa \\ ${ }^{3}$ Division of Neuropsychiatry, Department of Psychiatry, University of Cape Town and Groote Schuur Hospital, Cape Town, South Africa
}

Corresponding author: H M Cross (helenmargot@gmail.com)

\begin{abstract}
Background. The human immunodeficiency virus (HIV) can give rise to a spectrum of neuropsychological impairments known collectively as HIV-associated neurocognitive disorders (HAND). Although antiretroviral therapy (ART) has reduced the incidence of HIV dementia, the prevalence of milder forms of HAND has increased. It has been postulated that incomplete central nervous system (CNS) viral suppression or potential drug toxicity, both of which could be related to the CNS penetration effectiveness (CPE) of ART regimens, may contribute to this phenomenon.

Objective. This study compared cognitive outcomes in clade C-infected HIV patients in South Africa treated for 1 year with ART regimens with differing CPE scores.

Methods. We assessed 111 HIV-positive patients with varying levels of cognitive function at baseline (pre-ART) and then a year later. A neuropsychological battery was administered at both visits to derive global deficit scores. ART regimen data were collected at the follow-up visit. Some participants remained ART-naïve during this period, thus providing a non-treatment control group.

Results. Significantly more ART recipients maintained or improved cognitive function compared with patients not on ART ( $p=0.017)$. There was no significant difference in cognitive outcomes between higher and lower CPE regimen groups $(p=0.473)$.

Conclusions. ART preserves or improves cognition in HIV-infected patients after 1 year, irrespective of the regimen's CPE. South Africa's current low CPE-scored first-line regimen performed as well as higher CPE-scored regimens. These findings are reassuring for South Africa, but larger, longer-term studies would be more definitive.
\end{abstract}

S Afr Med J 2013;103(10):758-762. DOI:10.7196/SAMJ.6677

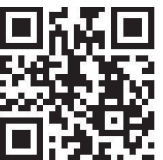

The human immunodeficiency virus (HIV) is thought to invade the central nervous system (CNS) early in the course of infection. Infected individuals may develop cognitive impairment of varying severity, ranging from mild deficits evident only on formal cognitive testing to severe HIV-associated dementia (HIV-D). This spectrum of disease is known collectively as HIV-associated neurocognitive disorders (HAND). ${ }^{[1]}$

HAND is highly prevalent: it is estimated that $30-60 \%$ of HIVpositive individuals are affected. ${ }^{[2]}$ The resulting cognitive impairment can interfere with social and occupational functioning, and affect adherence to antiretroviral therapy (ART). ${ }^{[3]}$ With the success of ART and now much longer life expectancy for HIV-infected persons, treatment of HIV has shifted from palliation to chronic disease management. Functional outcomes and quality of life concerns, including cognitive ability, have become all the more important. ${ }^{[4]}$

The majority of the worldwide HIV burden lies in sub-Saharan Africa. Clade C is the predominant HIV-1 subtype in South Africa. ${ }^{[5]}$ Only a few studies have been done in distinct clade $\mathrm{C}$ cohorts, and these suggest a high risk of HAND. ${ }^{[6,7]}$

Since the introduction of ART, the incidence of HIV-D has decreased in the developed world from approximately $16 \%$ of HIV-positive patients being affected to less than $5 \% .{ }^{[8-10]}$ ART typically produces a significant improvement in neurocognitive status within 6 months of treatment. ${ }^{[11]}$ This does not, however, imply a complete recovery in all cases. In fact, incident cases of cognitive dysfunction can still arise while patients are on suppressive ART regimens. Prevalence rates of the milder forms of HAND may even have increased in the era of ART. ${ }^{[10,12,13]}$ This is partly attributable to longer survival (with longer overall exposure to the neurological effects of HIV), and to increased awareness and diagnosis of HAND. However, it may also relate to incomplete control of the virus and its effects on the CNS, and possibly to neurotoxicity of the antiretrovirals themselves. ${ }^{[14]}$

One of the proposed reasons for the continually high incidence of HAND in the ART era relates to the difficulty posed by the bloodbrain barrier $(\mathrm{BBB})$ for the passage of drugs into the CNS. Although previously investigated by many groups, a CNS penetration effectiveness (CPE) ranking system was formally proposed by Letendre et al. ${ }^{[15]}$ in 2008 and updated in 2010. ${ }^{[16]}$ This system ranks different antiretroviral agents according to their ability to cross the BBB and achieve control of HIV replication in the CNS. Each antiretroviral agent was assigned a score according to its chemical properties, cerebrospinal fluid (CSF) plasma concentrations, and effectiveness in achieving CNS HIV control ( 1 = below average; 2 = average; $3=$ above average; $4=$ much above average). A regimen score can be calculated by adding the individual agent scores. A regimen with a total score of $\leq 7$ is considered to have low penetration in the CNS, while a score of $>7$ is considered to be highly penetrant. ${ }^{[17]}$ There is convincing evidence that this system correctly identifies regimens that will achieve control of HIV replication in the CNS, i.e. regimens with higher CPE have been shown to be associated with CSF viral load (VL) suppression. ${ }^{[15]}$ However, studies looking at the correlation between cognitive outcomes and 
higher CPE regimens have shown mixed results. ${ }^{[18-22]}$ Tozzi et al. ${ }^{[18]}$ and Letendre et al. ${ }^{[2]}$ both found an association between higher CPE regimens and improved neuropsychological function, while Rourke et al. ${ }^{[19]}$ and Ciccarelli et al. ${ }^{[20]}$ found no association between CPE scores and cognitive outcomes. Marra et al. ${ }^{[22]}$ found an association between higher CPE scores and poorer cognitive performance, which they suggested may relate to increased drug toxicity given the increased CNS penetration.

ART only became readily available in South Africa's public sector in 2003 (as opposed to 1996 in the developed world), and access remains limited in some areas. Additionally, until very recently national guidelines allowed that the majority of patients be started on ART only when their CD4 count fell below 200 cells/ $\mu l^{\left[{ }^{[23]}\right.}$ This is in contrast to the 350 cells/ $\mu$ ladvised by the World Health Organization (WHO), ${ }^{[24]}$ and to which South African guidelines were amended in August 2011. ${ }^{[25]}$ If a diagnosis of HIV-D is made, the patient can be classified as WHO disease stage IV, and will then be considered for ART regardless of $\mathrm{CD} 4$ count. However, no provision is currently made for the milder forms of HAND. The fact that the South African ART threshold was a CD4 count less than 200 cells/ $\mu$ l for so long could have resulted in a higher prevalence of HAND in our setting, given that more advanced immunosuppression has been related to an increased risk of HAND. ${ }^{[10]}$

In addition to the heavy burden of HIV disease, South Africa is a resource-poor setting. This equates to busy, often underresourced clinics, where there is minimal time for the lengthy patient consultations that may be necessary to detect the subtler forms of HAND. Furthermore, as a result of protocol-driven management in the public sector, we have limited scope for individualised ARV choices.

Of note is the change in the South African Antiretroviral Guidelines in April 2010. ${ }^{[23]}$ This change was largely aimed at discontinuing the use of the agent stavudine, and replacing it with tenofovir. Although the new first-line regimen is better tolerated, it has a lower CPE ranking ( 7 or 8 previously, compared with 6 or 7 currently), which may be significant in terms of cognitive outcomes in HIV. Concerns had been raised that widespread use of a lower CPE regimen might increase the incidence of HAND at a population level.

Also of interest is that the first-line regimen for pregnant women, zidovudine, lamivudine and nevirapine, has a CPE ranking of 10 . This regimen would then be continued after pregnancy unless unacceptable side-effects or treatment failure necessitated a change. These two main regimens currently in use in South Africa create an interesting split of HIV-positive patients using high- and low-ranking CPE regimens.

The recent change in the South African first-line ART regimen from a higher to a lower CPE regimen, as well as the existence of the group of HIV-positive patients on the pregnancy regimen, allowed examination of the difference in cognitive outcomes according to ART regimen CPE in an HIV-1 clade C-specific cohort.

\section{Objective}

To examine the effect of ART regimen CPE on cognitive function in clade C-infected HIV-positive people. We did this by comparing cognitive outcomes between the group receiving the pre-April 2010 ART regimen and the pregnancy regimen (both higher $\mathrm{CPE}$ ) and the post-April 2010 lower CPE ART regimen. We hypothesised that patients receiving the higher CPE regimens would have better cognitive outcomes after 1 year.

\section{Methods}

This study formed part of a collaborative research programme into HIV-associated neurocognitive disorders in the Western Cape Province of South Africa by the departments of Psychiatry, Neurology and
Geriatric Medicine at Groote Schuur Hospital/University of Cape Town. The research project was approved by the Human Research Ethics Committee of the Faculty of Health Sciences at the University of Cape Town (REC 263/2007, amendment 19 May 2008, and REC 203/2008).

HIV-positive participants attending primary level ARV clinics for highly active antiretroviral therapy initiation were recruited from Nolungile Clinic in Site C, Khayelitsha, Woodstock Community Health Centre and Mitchells Plain Community Health Centre. HIVnegative control participants, recruited from voluntary counselling and testing centres in the same areas, were included to assess the neuropsychological norms for the population.

Entry criteria included being between the ages of 18 and 35 years and having completed 7 years of schooling. Participants with uncontrolled medical conditions, other psychiatric or neurological disorders, substance or alcohol abuse or previous moderate to severe head injury were excluded.

The participants were assessed initially (pre-ART) and followed up approximately a year later. A neuropsychological test battery was administered at both visits to derive two sets of global deficit scores (GDSs). ${ }^{[26,27]}$

HIV-negative, demographically similar, control participants $(N=103)$ underwent testing with a full neuropsychological battery, encompassing several different domains of cognitive function. This created a set of 'normal' raw scores for each test, which allowed computation of the 'normal' mean and standard deviation for each test in the specific study population. Summary scores for each ability domain were calculated by adding the individual standard scores for each test covering that domain, and dividing by the number of such tests. The specific scores used for each domain were: non-dominant hand scores for the finger tapping test and grooved pegboard test (motor), Hopkins Verbal Learning Test recall and Brief Visuospatial Memory Test recall scores (learning), Mental Alternation Test and Wechsler Memory Scale III mental control scores (attention), Digit Symbol Coding, Trail Making Test A and Colour Trails I (processing speed), Colour Trails II, Stroop Colour Word, Wisconsin Card Sorting Test perseverative errors and Rey-Osterrieth Complex Figure copy scores (executive function), and animal and fruit/vegetable fluency scores (verbal). This 'summary $Z$-score' for each domain was then converted to a $t$-score, and $t$-scores were converted to deficit scores according to the schema proposed by Heaton et al. ${ }^{[26]}$ The GDS was calculated by adding the deficit scores from each domain, and dividing by the total number of added scores.

Although the cut-point of $\geq 0.50$ has been shown to have the best diagnostic accuracy in terms of impairment, ${ }^{[27]}$ different cut-points could be used. By definition, a GDS of 0 implies normality, while larger numbers imply worsening neuropsychological impairment.

For the purpose of this study, the participants were grouped into three categories of neuropsychological impairment: normal (GDS $<0.25)$, mild-moderate impairment $(0.25 \leq \mathrm{GDS}<0.75)$ and severe impairment (GDS $\geq 0.75$ ). This approach was developed by Joska et al., ${ }^{[28]}$ and the three groups were shown to correlate moderately well with the HAND diagnoses as defined by Antinori et al.$^{[1]}$ of normal function, asymptomatic neurocognitive impairment/mild neurocognitive disorder (as these essentially have the same degree of neuropsychological impairment, but differ on functional status) and HIV-D. Using this approach instead of the defined HAND categories removes the need to undertake an assessment of activities of daily living and related functional impairment.

Details regarding ART (including regimen, duration and virological response) were collected at the follow-up visit. Participants were excluded if they had not been on one consistent ART regimen for at least 10 months, and if they were not virally suppressed on 
their ART regimens. Those meeting the ART requirements had CPE scores assigned to their regimens. A small number of participants were found still to be ART-naive a year later, and were used as unintended controls to examine the effect of exposure to ART.

Data were analysed using STATA (version 11). The level of statistical significance was set at $p=0.05$. Distributions of the variables were determined using the Shapiro-Wilk test, and then parametric or nonparametric methods used as appropriate. Analysis of variance (ANOVA) and the Wilcoxon ranksum and Pearson chi-square tests were used for the analyses. ANOVA tests were done to compare the baseline characteristics of the cognitive function groups. Pearson chi-square tests were used to compare the change in cognitive function category, firstly between the ART and no-ART groups, and secondly for the higher CPE versus lower CPE groups. Wilcoxon rank-sum tests were used to compare these changes as a continuous variable, using the actual GDS score change.

\section{Results}

We obtained complete data on 111 participants, of whom 89 had been initiated on ART and 22 had not; 69 of the 89 met the ART requirements (listed above) to be included in the CPE analysis. Of these 69, 31 were classified into the low-regimen $\mathrm{CPE}$ category, while 38 were in the high-regimen CPE category (Fig. 1).

All those initiated on ART had a CD4 count below 350 cells/ $\mu$. For this reason 2 of the ART-naïve participants were excluded from the analysis because their CD4 counts were above 350 cells $/ \mu$ l, making them not comparable to the ART group.

The baseline characteristics of the cognitive function groups showed no significant difference in terms of CD4 count and most demographic features, with the exception of level of education $(p \leq 0.001)$. The groups that did and did not receive ART were similar in terms of baseline CD4 count (mean 162.32 v. 181.15 cells $/ \mu \mathrm{l}), t$-test (df $86, t=-1.10, p=0.275$ ) and GDS (median 0.27 v. 0.33 , Wilcoxon rank-sum test: $Z=-0.154$, $p=0.878$ ). Table 1 shows the breakdown of the cognitive function groups according to ART and CPE category.

To analyse the change in cognitive function with respect to ART status, we analysed how many of the participants maintained or improved cognition, and how many worsened. This was done by comparing the follow-up GDS with the baseline GDS.

Of the ART group, 56 (81.2\%) maintained or improved cognitive function, while 13 $(18.8 \%)$ worsened. Of the non-ART group,

Table 1. Breakdown of cognitive function groups according to ART and CPE category

\begin{tabular}{|c|c|c|c|c|}
\hline \multirow[b]{3}{*}{ Baseline cognitive function group } & \multicolumn{4}{|c|}{$n$} \\
\hline & \multicolumn{2}{|c|}{ ART category } & \multicolumn{2}{|c|}{ CPE category } \\
\hline & ART & Non-ART & Low CP & High CPE \\
\hline Normal cognitive function, GDS $<0.25$ & 33 & 8 & 15 & 18 \\
\hline Mild-moderate impairment, $0.25 \leq \mathrm{GDS}<0.75$ & 21 & 9 & 10 & 11 \\
\hline Severe impairment, GDS $\geq 0.75$ & 15 & 3 & 6 & 9 \\
\hline
\end{tabular}

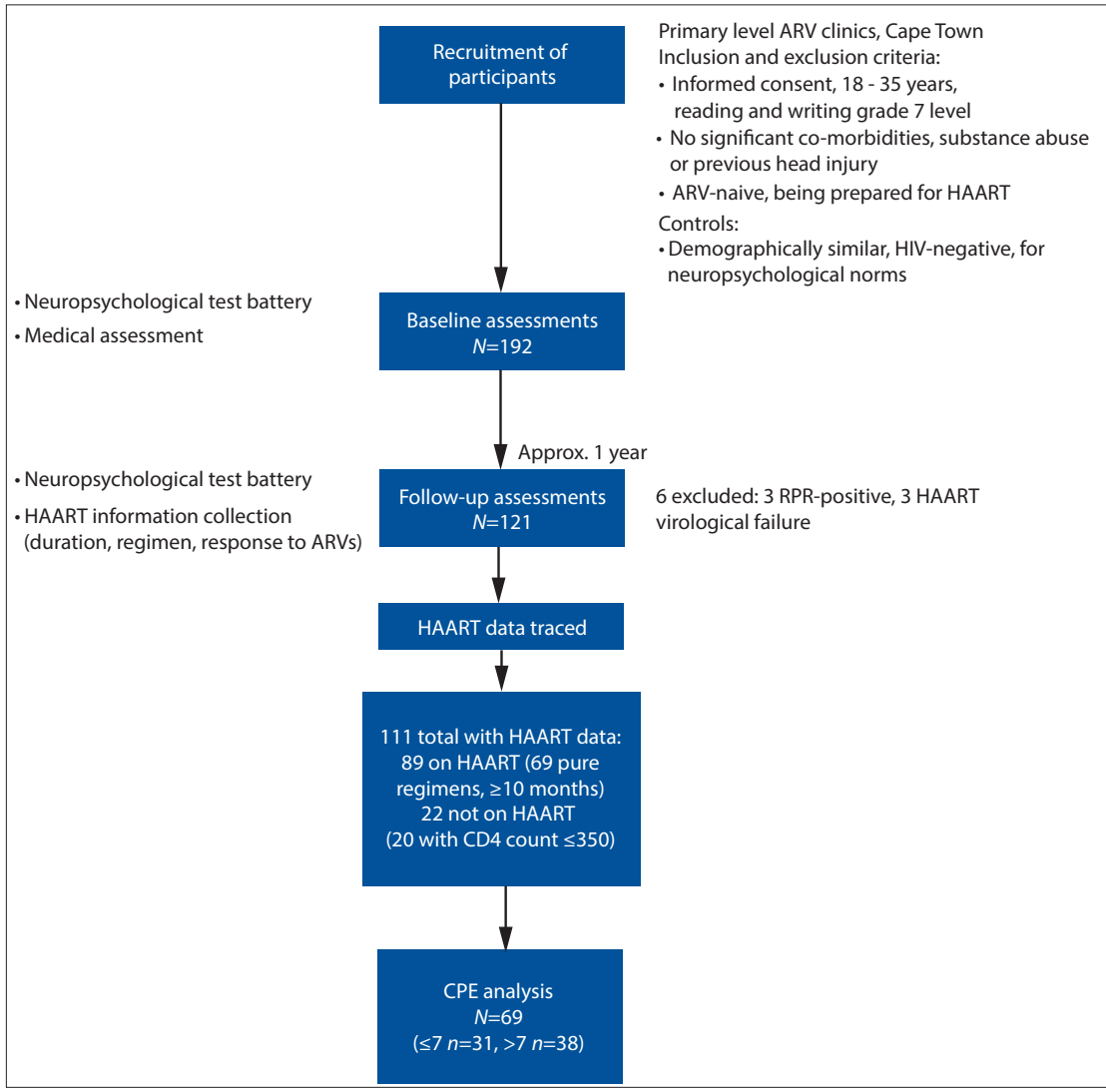

Fig. 1. Flow diagram of the study process (ARV = antiretroviral; HAART = highly active antiretroviral therapy; HIV = human immunodeficiency virus; $R P R=$ rapid plasma reagin; $C P E=$ central nervous system penetration effectiveness).

$11(55.0 \%)$ maintained or improved cognitive function, while 9 (45.0\%) deteriorated. There was a significant difference in cognitive response between the group that had received ART and the group that had not (Pearson's chi-square test: $\left.\chi^{2}(1, n=89)=5.70, p=0.017\right)$. More of those patients who had received ART than those who had not, maintained or improved cognitive function (Fig. 2). The actual GDS change was also examined as a continuous variable between the two groups (ART group -0.07, non-ART group 0). The ART group had a greater GDS improvement (Wilcoxon rank-sum test: $Z=-2.09, p=0.036$ ).

Of the high CPE group, 32 (84.2\%) maintained or improved cognitive function, while $6(15.8 \%)$ worsened. Of the low $\mathrm{CPE}$ group, 24 (77.4\%) maintained or improved cognitive function, while 7 (22.6\%) deteriorated (Fig. 3). There was no significant difference in cognitive response between the higher and lower CPE regimen groups at the 1-year follow-up visit (Pearson's chi-square test: $\chi^{2}(1, n=69)=0.51, p=0.473$.) Similarly, the median GDS changes in the high CPE group (-0.13) and the low CPE group (0) were not significantly different (Wilcoxon rank-sum test: $Z=0.99, p=0.320$ ).

\section{Discussion}

To our knowledge, this is the first published study from South Africa reporting on neurocognitive outcomes in patients receiving low and high $\mathrm{CPE}$ scoring ART 


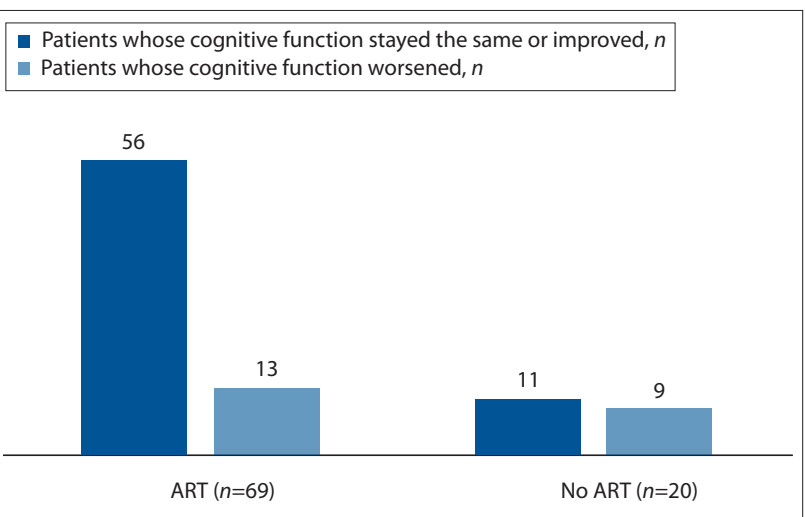

Fig. 2. Cognitive response after 1 year according to antiretroviral therapy (ART) status.

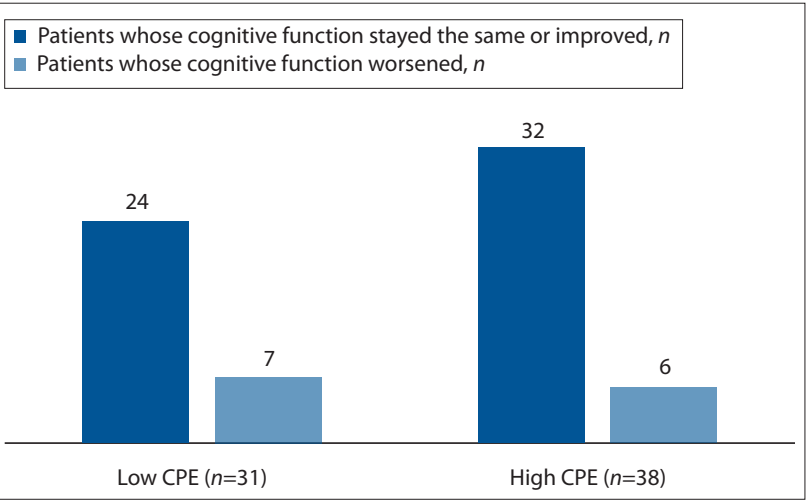

Fig. 3. Cognitive response after 1 year according to highly active antiretroviral therapy regimen central nervous system penetration effectiveness (CPE) group.

regimens. We noted that ART was beneficial in preserving, and in some cases improving, cognitive function in patients with CD4 counts of $\leq 350$ cells $/ \mu$ l. We found no association between the CPE of the ART regimen used and cognitive outcome after 1 year, i.e. cognitive outcomes did not differ between participants treated with the previous first-line regimen (CPE 7/8), the new first-line regimen (CPE 6/7) or the regimen initiated during pregnancy (CPE 10).

Although there is convincing evidence that the CPE system correctly identifies regimens that will achieve control of HIV replication in the $\mathrm{CNS},{ }^{[15,22]}$ studies looking at the correlation between cognitive outcomes and higher CPE regimens have shown mixed results.

Tozzi et al. ${ }^{[18]}$ and Letendre et al. ${ }^{[21]}$ both found an association between higher CPE regimens and improved neuropsychological function. This finding was expected, given the improved CSF VL control. However, Rourke et al. ${ }^{[19]}$ and Ciccarelli et al. ${ }^{[20]}$ found no association between CPE scores and cognitive outcomes. In this regard it is important to remember that CSF VL is not necessarily equivalent to brain VL. Postmortem studies have shown higher levels of HIV in brain tissue than in the CSF of the same patients. ${ }^{[29]}$

Marra et al. ${ }^{[22]}$ found an association between higher CPE scores and poorer cognitive performance, which they suggested may relate to increased drug toxicity given the increased CNS penetration. ART itself may have CNS neurotoxic properties, especially given the welldescribed toxic effects on peripheral nerves. Neurochemical studies have shown that almost all antiretroviral agents induce some degree of neuronal dysfunction. ${ }^{[30,31]}$ Clinical studies have also suggested potential ART neurotoxicity. For example, Robertson et al. ${ }^{[14]}$ showed significant improvement in cognitive function in a cohort of patients taken off ART.

There are many possible reasons for the conflicting literature regarding cognitive changes on higher $\mathrm{CPE}$ regimens. These include differing study designs, differing methods of assessing and classifying neuropsychological function, and small sample sizes. Study numbers range from 31 (Letendre et al..$^{[21]}$ ) to 255 (Rourke et al. ${ }^{[19]}$ ). Letendre et al. ${ }^{[21]}$ also noted that neuropsychological improvement was greater in ART-naïve participants, possibly because participants on ART accumulated resistant mutations. Some of the above studies have been done using ART-naïve participants, ${ }^{[18,19]}$ and others naïve and experienced participants..$^{[20-22]}$

Not all studies measured both CSF VL response and cognitive outcomes. Tozzi et al. ${ }^{[18]}$ noted that the correlation between higher CPE score and neuropsychological improvement was exclusive to those who achieved CSF VL suppression. This suggests that VL suppression rather than ART regimen was the key factor. Higher CPE regimens do, however, usually achieve greater CSF VL suppression.

Our findings are consistent with international studies that also showed no association between ART regimen CPE and cognitive outcomes. ${ }^{[19,20]}$ However, as the literature shows, further research is still required in this area.

\section{Study limitations}

There was a relatively small number of participants for the CPE analysis, and a short follow-up. However, 1 year is not an unreasonable follow-up period, given that it has been shown that significant cognitive improvement is usually seen within 6 months of starting ART. ${ }^{[11]}$ It could be argued that a selection bias exists in this study, in terms of excluding those participants not on suppressive ART regimens for $\geq 10$ months. These participants may have had poorer cognitive function and therefore greater difficulty in accessing and adhering to ART. However, this study was primarily aimed at comparing different ART regimens, and these exclusions were therefore necessary. It was also unfortunate that CSF testing could not form part of this study. We did not have ethics approval to perform lumbar punctures, as the participants were well outpatients who did not require this test as part of their clinical management.

Our findings do, nevertheless, suggest that the new lower CPE-ranking first-line ART regimen may be as neuroprotective as the previous higher penetration regimen. We did not find an increased prevalence of HAND in the lower CPE regimen group at 1 year. We do not yet know whether these neuropsychological improvements will persist beyond a year, or what the impact will be on daily function. Ideally a larger study should be conducted, with both CSF and neuropsychological testing and a longer follow-up period of several years.

\section{References}

1. Antinori A, Arendt G, Becker JT, et al. Updated research nosology for HIV-associated neurocognitive disorders. Neurology 2007;69(18):1789-1799. [http://dx.doi.org/10.1212/01.WNL.0000287431.88658.8b]

2. Grant I. Neurocognitive disturbances in HIV. Int Rev Psychiatry 2008;20(1):33-47. [http://dx.doi. org/10.1080/09540260701877894]

3. Heaton K, Marcotte TD, Mindt MR, et al. The impact of HIV-associated neuropsychological impairment on everyday functioning. J Int Neuropsychol Soc 2004;10(3):317-331. [http://dx.doi. org/10.10170S1355617704102130]

4. Rackstraw S. HIV-related neurocognitive impairment - a review. Psychology, Health \& Medicine 2011;16(5):548-563. [http://dx.doi.org/10.1080/13548506.2011.579992]

5. Orrell C, Walensky RP, Losina E, Pitt J, Freedberg KA, Wood R. HIV type-1 clade C resistance genotypes in treatment-naïve patients after first virological failure in a large community antiretroviral treatment programme. Antivir Ther 2009;14(4):523-531.

6. Joska JA, Westgarth-Taylor J, Myer L, et al. Characterization of HIV-associated neurocognitive disorders among individuals starting antiretroviral therapy in South Africa. AIDS Behav 2011;15(6):1197-1203 [http://dx.doi.org/10.1007/s10461-010-9744-6]

7. Yepthomi T, Paul R, Vallabhaneni $S$, et al. Neurocognitive consequences of HIV in Southern India:

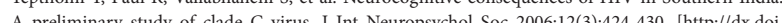
org/10.10170S1355617706060516 
8. Heaton RK, Franklin DR, Ellis RJ, et al. HIV-associated neurocognitive disorders before and during he era of combination antiretroviral therapy: Differences in rates, nature, and predictors. I Neuroviro 2011;17(1):3-16. [http://dx.doi.org/10.1007/s13365-010-0006-1]

9. McArthur J, Hoover D, Bacellar H, et al. Dementia in AIDS patients: Incidence and risk factors. Neurology 1993;43(11):2245-2252. [http://dx.doi.org/10.1212/WNL.43.11.2245]

10. Heaton RK, Clifford DB, Franklin JR, et al. HIV-associated neurocognitive disorders persist in the era of potent antiretroviral therapy: The CHARTER study. Neurology 2010;75(23):2087-2096. [http://dx.doi. rg/10.1212/WNL.0b013e318200d727]

11. Joska JA, Gouse H, Paul RH, Stein D, Flisher AJ. Does highly active antiretroviral therapy improve neurocognitive function? A systematic review. I Neurovirol 2010;16(2):101-114. [http://dx.doi. org/10.3109/13550281003682513]

12. Robertson KR, Smurzynski M, Parsons $\mathrm{TD}$, et al. The prevalence and incidence of neurocognitive impairme in the HAART era. AIDS 2007;21(14):1915-1921. [http://dx.doi.org/10.1097/QAD.0b013e32828e4e27]

13. Simioni S, Cavassini M, Annoni J, et al. Cognitive dysfunction in HIV patients despite long-standing suppression of viremia. AIDS 2010;24(9):1243-1250. [http://dx.doi.org/10.1097/QAD.0b013e3283354a7b]

14. Robertson KR, Su Z, Margolis DM, et al. Neurocognitive effects of treatment interruption in stable HIVpositive patients in an observational cohort. Neurology 2010;74(16):1260-1266. [http://dx.doi.org/10.1212/ WNL.0b013e3181d9ed09]

15. Letendre S, Marquie-Beck J, Capparelli E, et al. Validation of the CNS penetration-effectiveness rank for quantifying antiretroviral penetration into the central nervous system. Arch Neurol 2008;65(1):65-70. (htt://dx.doi.org/10.1001/archneurol.2007.31

6. Letendre S, Fitzsimons $C$, Ellis $R$, et al. Revised central nervous system penetration-effectiveness ranking. Presented at the 17th Conference on Retroviruses and Opportunistic Infections, $16-19$ February 2010, San
Pan Francisco, USA. Abstract 172

17. Letendre S. The CPE score (Lecture). Presented at the 4th International Meeting on HIV Infection and the Central Nervous System, 17-20 July 2011, Rome, Italy.

18. Tozzi V, Balestra P, Salvatori M, et al. Higher CPE score improved cognition, changes in cognition during antiretrovial therapy: Comparison of 2 different ranking systems to measure antiretroviral drug efficacy on HIV-associated neurocognitive disorders. J Acquir Immune Defic Syndr 2009;52(1):56-63. [http://dx.doi. org/10.1097/QAL.0b013e3181af83d6]

19. Rourke SB, Carvalhal A, Zipursky AR, et al. Examining the impact of CNS penetration-effectiveness of combination antiretroviral treatment (cART) on neuropsychological outcomes in persons living with HIV: Findings from the Ontario HIV Treatment Network (OHTN) cohort study. Presented at the 6th International AIDS Society Conference on HIV Pathogenesis, Treatment and Prevention, 17-20 July 2011, Rome, Italy. Abstract MOAB0104. http://pag ias2011.org/Abstracts.aspx?AID=2810 (accessed 2 April 2013).
20. Ciccarelli N, Fabbiani N, Di Giambenedetto S, et al. Efavirenz associated with cognitive disorders in otherwise asymptomatic HIV-infected patients. Neurology 2011;76(16):1403-1409. [http://dx.doi. org/10.1212/WNL.0b013e31821670fb]

21. Letendre SL, McCutchan JA, Childers ME, Woods SP, Lazzaretto D, Heaton RK. Enhancin antiretroviral therapy for human immunodeficiency virus cognitive disorders. Ann Neurol 2004:56(3):416-423. [http///dx doi.org/10.1002/ana.20198]

22. Marra CM, Zhao Y, Clifford DB, et al. Impact of combination antiretroviral therapy on cerebrospin fluid HIV RNA and neurocognitive performance. AIDS 2009;23(11):1359-1366. [http://dx.doi ro 10.1097/QAD.0b013e32832c4152]

23. South African Antiretroviral Treatment Guidelines 2010. http://www.uj.ac.za/EN/CorporateServices/ ioha/Documentation/Documents/ART\%20Guideline.pdf (accessed 4 April 2013).

24. World Health Organization. Antiretroviral Therapy for HIV Infection in Adults and Adolescents: Recommendations for a Public Health Approach. 2010 edition. http://whqlibdoc.who.int publications/2010/9789241599764_eng.pdf (accessed 4 April 2013).

25. South African National AIDS Council. Statement on the Meeting of the South African National AIDS Council (SANAC). 2011. http://www.sanac.org.za/files/uploaded/886_Plenary (accessed 4 April 2013).

26. Heaton RK, Grant I, Butters N, et al. The HNRC 500 - Neuropsychology of HIV infection at differen disease stages. I Int Neuropsychol Soc 1995; 1(3):231-251. [http://dx.doi.org/10.1017/S1355617700000230]

27. Carey CL, Woods SP, Gonzalez R, et al. Predictive value of global deficit scores in detecting Crever neuropsychological impairment in HIV infection. J Clin Exp Neuropsychol 2004:26(3):307-319.

28. Joska IA, Westgarth-Taylor J Hoare $J$ et al. Neuropsychological outcomes in adults commencing highly active anti-retroviral treatment in South Africa: A prospective study. BMC Infect Dis 2012;12(Feb):39. [http://dx.doi.org/10.1186/1471-2334/12/39]

29. Kumar A, Borodowsky I, Fernandez B, Gonzalez L, Kumar M. Human immunodeficiency virus type 1 RNA levels in different regions of human brain: Quantification using real-time reverse transcriptase-polymerase chain reaction. J Neurovirol 2007;13(3):210-224. [http://dx.doi.org/10.1080/13550280701327038]

30. Liner J, Robertson K, Meeker RB. Antiretroviral neurotoxicity. Poster presentation at the 39th Annual Neuroscience Meeting, 17-21 October 2009, Chicago, USA

31. Schweinsburg BC, Taylor MJ, Alhassoon OM, et al. Brain mitochondrial injury in human immunodeficiency virus-seropositive $(\mathrm{HIV}+)$ individuals taking nucleoside reverse transcriptase inhibitors. J Neurovirol 2005;11(4):356-364. [http://dx.doi.org/10.1080/13550280591002342]

Accepted 14 March 2013 